\title{
Rugosidade superficial do solo sob diferentes doses de resíduo de milho submetido à chuva simulada
}

\author{
Ildegardis Bertol(1), Antonio Paz González ${ }^{(2)}$ e Eva Vidal Vázquez ${ }^{(2)}$
}

\begin{abstract}
(1)Universidade do Estado de Santa Catarina, Centro de Ciências Agroveterinárias, Dep. de Solos, Caixa Postal 281, CEP 88520-000 Lages, SC. E-mail: a2ib@cav.udesc.br (2)Universidad de A Coruña, Área de Edafología y Química Agrícola, Facultad de Ciencias, Campus Zapateira, s/no 15071 - La Coruña, Espanha. E-mail: tucho@udc.es, evavidal@lugo.uds.es
\end{abstract}

Resumo - O objetivo deste trabalho foi avaliar a rugosidade e a tortuosidade da superfície do solo, preparado com diferentes doses de resíduo cultural de milho, e relacioná-las com o volume de chuva simulada. Em um Cambissolo, em La Coruña, Espanha, em agosto de 2005, avaliou-se a rugosidade superficial, no preparo mínimo sob chuva simulada, com as doses de $0,2,4,6$ e $8 \mathrm{t} \mathrm{ha}^{-1}$ de resíduo de milho picado e semi-incorporado ao solo. Aplicaram-se oito testes de chuva simulada, com $65 \mathrm{~mm} \mathrm{~h}^{-1}$ e $60 \mathrm{~min}$ cada um. A rugosidade e a tortuosidade, ao acaso, foram menores do que na condição linear e, as desta condição foram menores do que na condição original. A redução da rugosidade e da tortuosidade, em decorrência das chuvas, diminuiu com o aumento da dose de resíduo de milho. A rugosidade superficial foi mais fortemente influenciada quando se eliminou o efeito da declividade do terreno do que quando se eliminaram, simultaneamente, os efeitos da declividade e das marcas de preparo do solo para o seu cálculo.

Termos para indexação: índice de rugosidade, microrrelevo superficial, erosão hídrica.

\section{Soil surface roughness with different doses of corn residue submitted to simulated rainfall}

\begin{abstract}
The objective of this work was to determine the surface roughness and the tortuosity of the soil tilled with different doses of corn residues, and to relate them to simulated rainfall volume. In an Inceptisol, in La Coruña, Spain, in August of 2005, the surface roughness and tortuosity were evaluated in the minimum soil tillage system under simulated rain, with the doses $0,2,4,6$, and $8 \mathrm{tha}^{-1}$ of corn residues semi-incorporated to the soil for manual tillage. Eight tests of simulated rain were applied, with $65 \mathrm{~mm} \mathrm{~h}^{-1}$ and $60 \mathrm{~min}$ each one. The surface roughness and tortuosity were determined before the application of the residue, immediately after the soil tillage and immediately after the $1^{\text {st }}, 2^{\text {nd }}, 4^{\text {th }}, 6^{\text {th }}$ and $8^{\text {th }}$ tests of the rain. The reduction of the roughness and of the tortuosity, due to rain, decreased with the increase of corn residues doses. The superficial roughness was more strongly influenced, when the effect of land steepness was eliminated, than when were eliminated, simultaneously, the effects of steepness and of tillage marks for this calculate.
\end{abstract}

Index terms: roughness index, surface microrelief, water erosion.

\section{Introdução}

A rugosidade superficial do solo constitui o microrrelevo e se refere ao enrugamento da superfície, ocasionado por microelevações e microdepressões espacialmente distribuídas (Linden \& Van Doren Junior, 1986). O microrrelevo superficial é influenciado pelo preparo do solo (Bertol et al., 2006; Castro et al., 2006), que afeta o volume de poros da camada preparada e que, com a cobertura por resíduos vegetais, influencia a armazenagem de água na superfície (Onstad, 1984), a infiltração de água no solo, a velocidade e volume de enxurrada, a retenção de sedimentos (Darboux \&
Huang, 2005) e, conseqüentemente, as perdas de solo (Kamphorst et al., 2000; Bertol et al., 2006).

O microrrelevo superficial é dependente: do tipo e da intensidade de preparo, e do teor de água do solo antecedente ao preparo; da quantidade, tipo e manejo de resíduo vegetal existente no solo; e do tipo de solo (Römkens \& Wang, 1986; Kamphorst et al., 2000; Bertol et al., 2006; Castro et al., 2006). O decaimento da rugosidade é influenciado pelo volume e intensidade de chuvas e de enxurrada, e pelo tipo e manejo de solo (Paz González \& Castro, 1996; Eltz \& Norton, 1997; Bertol et al., 2006; Castro et al., 2006). Ainda, a rugosidade é afetada pela densidade e porosidade do 
solo (Lehrsch, et al., 1987), e pela estabilidade de agregados em água (Bertol et al., 2006), as quais dependem do manejo do solo e, por isso, influenciam a qualidade da estrutura e regulam a velocidade e o grau de sua degradação.

Entre os preparos de solo conservacionistas, o cultivo mínimo (combinação de escarificador e grade) normalmente ocasiona elevada rugosidade superficial, em consequiência dos tipos de equipamento utilizados, da conseqüente menor intensidade de mobilização do solo (Bertol et al., 1997, 2006; Castro et al., 2006), e dos resíduos vegetais que são semi-incorporados ao solo por este tipo de preparo. Assim, o cultivo mínimo apresenta maior rugosidade superficial do que o preparo convencional e, este, maior do que a semeadura direta e do que o solo sem cultivo.

A rugosidade ao acaso se constitui no mais antigo e tradicional índice que descreve o microrrelevo do solo e a distribuição casual dos torrões do solo, em uma superfície submetida a determinado manejo. $\mathrm{O}$ índice de rugosidade representa o intervalo vertical da rugosidade, sem levar em conta a distribuição espacial dos pontos altos e baixos no espaço do microrrelevo. Portanto, duas superfícies com o mesmo índice RR podem apresentar morfologias distintas e, conseqüentemente, diferentes capacidades para armazenar água na superfície (Huang \& Bradford, 1992; Kamphorst et al., 2000).

A tortuosidade ao acaso é outro indicador de rugosidade. O índice de tortuosidade descreve a relação entre a extensão do perfil de uma superfície de solo rugosa e a distância, entre os pontos inicial e final, da referida superfície. Portanto, este índice pode representar a capacidade de determinada superfície, para dificultar a enxurrada superficial. No entanto, o índice de tortuosidade depende da escala em que foram tomadas as medidas de rugosidade superficial (Skidmore, 1997). Assim, duas superfícies de solo com o mesmo índice de rugosidade, calculado com dados obtidos em escalas distintas, apresentarão índices de tortuosidade diferentes e, portanto, apresentarão diferentes capacidades para armazenar água e dificultar a enxurrada superficial (Kamphorst et al., 2000).

O objetivo deste trabalho foi quantificar a rugosidade e a tortuosidade da superfície do solo, preparado com diferentes doses de resíduo cultural de milho e relacioná-las com o volume de chuva simulada.

\section{Material e métodos}

O trabalho foi realizado no campo, entre março e agosto de 2005, na Estação Experimental de Pazo de Lóngora, na
Provincia de La Coruña, na Comunidade Autónoma de Galicia, Espanha. O solo no local do experimento foi classificado como Cambissolo e apresentava $121 \mathrm{~g} \mathrm{~kg}^{-1} \mathrm{de}$ areia grossa, $245 \mathrm{~g} \mathrm{~kg}^{-1}$ de areia fina, $146 \mathrm{~g} \mathrm{~kg}^{-1}$ de silte grosso, $218 \mathrm{~g} \mathrm{~kg}^{-1}$ de silte fino e $270 \mathrm{~g} \mathrm{~kg}^{-1}$ de argila, a $0-0,2$ m de profundidade. Nessa camada, o teor de carbono orgânico foi de $26,2 \mathrm{~g} \mathrm{~kg}^{-1}$, o nitrogênio mineral de $2,2 \mathrm{~g} \mathrm{~kg}^{-1}$ e o $\mathrm{pH}$ em água $(1: 1)$ de 6,34 . A declividade média do terreno foi de $0,06 \mathrm{~m} \mathrm{~m}^{-1}$ no local do experimento.

A sistematização da área experimental, em fevereiro de 2005, foi efetuada com duas operações de arado de aiveca a $0,2 \mathrm{~m}$ de profundidade, três operações de grade de disco a $0,12 \mathrm{~m}$ de profundidade, e uma operação de enxada rotativa a $0,08 \mathrm{~m}$ de profundidade. Sob essas condições, com rugosidade média, em março de 2005 foram instalados os tratamentos, sem repetição, em parcelas experimentais de 1x1 m, do seguinte modo: $\mathrm{T} 1$, sem resíduo cultural; T2, 2 t ha ${ }^{-1}$ de resíduo cultural de milho; $\mathrm{T} 3,4 \mathrm{t} \mathrm{ha}^{-1}$ de resíduo cultural de milho; $\mathrm{T} 4,6 \mathrm{t} \mathrm{ha}^{-1}$ de resíduo cultural de milho; e T5, 8 t ha $^{-1}$ de resíduo cultural de milho. O resíduo de milho foi picado em pedaços de cerca de $5 \mathrm{~cm}$ e distribuído uniformemente na superfície do solo. Procedeu-se, em seguida, à escarificação manual, com o objetivo de produzir sulcos na parcela e incorporar aproximadamente $30 \%$ da massa do resíduo vegetal. A escarificação foi feita com uma ripa de madeira pontiaguda, que simulou o trabalho de uma haste de escarificador, com cinco sulcos por parcela, à profundidade de $0,15 \mathrm{~m}$, no sentido da pendente do terreno. Após a operação de escarificação, a cobertura do solo foi a seguinte: ausente no T1; $0,48 \mathrm{~m}^{2} \mathrm{~m}^{-2}$ no T2; $0,70 \mathrm{~m}^{2} \mathrm{~m}^{-2}$ no T3; $0,80 \mathrm{~m}^{2} \mathrm{~m}^{-2}$ no T4; e $0,95 \mathrm{~m}^{2} \mathrm{~m}^{-2}$ no T5. A declividade da superfície do solo, no interior das parcelas experimentais, foi de $0,12 \mathrm{~m} \mathrm{~m}^{-1}$. Sobre os tratamentos, foram aplicados oito testes de chuva simulada; o primeiro foi imediatamente após a instalação dos tratamentos, e os demais em intervalos de tempo variáveis e suficientes para permitir a secagem da superfície do solo.

As chuvas simuladas foram de $65 \mathrm{~mm} \mathrm{~h}^{-1}$, com duração de uma hora, aplicadas por meio de um microssimulador de chuvas semelhante ao utilizado por Navas et al. (1990). A chuva foi propiciada por um único aspersor do tipo Fulljet 1/8 GG6SQ, fixo, com jato aproximadamente quadrado, que recobria uma área útil de $1,96 \mathrm{~m}^{2}$. O aspersor foi mantido a 2,30 $\mathrm{m}$ acima da superfície do solo. O intervalo de tempo, entre os testes de chuva simulada, variou de cinco a dez dias, assim, as chuvas foram aplicadas sobre solo seco. 
A rugosidade superficial do solo foi avaliada imediatamente após o preparo do solo e imediatamente após o primeiro, o segundo, o quarto, o sexto e o oitavo testes de chuva. Para efetuar as leituras do microrrelevo superficial, utilizou-se um rugosímetro com 20 varetas de alumínio, com $600 \mathrm{~mm}$ de comprimento e $8 \mathrm{~mm}$ de diâmetro cada uma, distanciadas $30 \mathrm{~mm}$ umas das outras e distribuídas ao longo de uma linha no suporte do rugosímetro, ao qual estava acoplada uma câmara fotográfica digital distanciada 1,80 m do conjunto de varetas. Isto possibilitou a tomada de uma fotografia do conjunto de varetas que refletiam as alturas da superfície do solo, localizadas transversalmente ao declive da parcela experimental. O conjunto de varetas foi movido sobre o suporte do rugosímetro, em 20 posições na direção da pendente da parcela, em distâncias de $30 \mathrm{~mm}$ uma das outras, para a tomada de 20 fotografias em cada parcela. A digitalização das fotografias permitiu a leitura de 400 pontos de altura da superfície do solo, no centro da parcela, numa área de amostragem de $360.000 \mathrm{~mm}^{2}$ (600x600 mm).

A rugosidade da superfície do solo foi calculada por meio de dois métodos, para obtenção do índice de rugosidade (RR) e do índice de tortuosidade (T). O índice de rugosidade foi calculado pelo método proposto por Kamphorst et al. (2000), tendo-se calculado o desvio-padrão das alturas, em vez do erro-padrão, e tendo-se utilizado os dados de altura da superfície do terreno, sem transformálos para logaritmo e sem eliminar os seus valores extremos. O índice de tortuosidade foi calculado conforme o método proposto por Kamphorst et al. (2000), tendo-se calculado a relação entre a extensão do perfil da superfície rugosa (considerando-se as ondulações superficiais do terreno) e a extensão da mesma superfície em linha reta (tendo-se desconsiderado as referidas ondulações). Os dois índices (RR e T) foram obtidos com os dados em três condições: foram mantidos no cálculo os efeitos da declividade do terreno e das marcas do preparo do solo (RR e T original); foi mantido no cálculo o efeito das marcas do preparo e retirado o efeito da declividade (RR e T linear); e foram retirados do cálculo, simultaneamente, os efeitos da declividade e das marcas do preparo (RR e $\mathrm{T}$ ao acaso).

Os dados de rugosidade e tortuosidade linear foram relacionados aos respectivos dados originais, e os dados de rugosidade e tortuosidade ao acaso foram relacionados aos respectivos dados lineares, pelo modelo de regressão linear simples. Foram feitas relações entre os índices de rugosidade e de tortuosidade ao acaso e o volume de chuvas, pelo modelo exponencial do tipo $\mathrm{y}=\mathrm{a} e^{-\mathrm{bx}}$.

\section{Resultados e discussão}

A rugosidade ao acaso da superfície, após o preparo, foi média, em relação à obtida por Eltz \& Norton (1997), Bertol et al. (2006) e Castro et al. (2006) e, também, em relação à escala de valores de rugosidade estabelecida por Renard et al. (1997) para a RUSLE (Tabela 1). Isto se justifica, porque o preparo do solo ocasionou torrões e microdepressões de dimensões médias.

O preparo efetuado sobre o solo com o resíduo de milho aumentou a rugosidade ao acaso em 2,9 vezes, em relação à rugosidade anterior ao preparo, na média dos tratamentos, ou seja, de 6,4 mm, antes do preparo, para 18,4 mm, após o preparo (Tabela 1). O aumento de $12 \mathrm{~mm}$ representa o efetivo incremento da capacidade do solo e do resíduo vegetal para armazenar água na superfície. No solo sem resíduo (T1), o aumento da rugosidade ao acaso pelo preparo foi de 5,9 vezes, em relação à rugosidade anterior ao preparo, enquanto na média dos demais tratamentos (com resíduo), o aumento dessa rugosidade foi de 2,4 vezes. Assim, as peças do resíduo não incorporado ao solo, pelo preparo, diminuíram a rugosidade superficial nos tratamentos com resíduo, em relação ao tratamento sem resíduo, já que as mesmas peças foram alojadas, principalmente, nas depressões do microrrelevo, tendo rebaixado sua profundidade média. Este efeito foi mais pronunciado no tratamento com a maior dose de resíduo aplicado ao solo (T5).

A rugosidade do solo calculada com os dados originais, obtidos imediatamente após o preparo, foi 2,4 vezes maior do que a rugosidade ao acaso, na média dos tratamentos (Tabela 1). Estes dados concordam com os obtidos por Eltz \& Norton (1997) e Bertol et al. (2006), e mostram o efeito negativo do declive do terreno de $12 \%$, e das marcas do preparo do solo, na rugosidade ao acaso, a qual representa a efetiva capacidade da superfície do solo para armazenar água (Onstad, 1984).

Ao se retirar o efeito do declive do terreno, no cálculo da rugosidade superficial, o valor desta se manteve ainda $35 \%$ maior do que o da rugosidade ao acaso, na média das chuvas e dos tratamentos (Tabela 1). Isto demonstra que as marcas do preparo do solo tiveram maior efeito no aumento da rugosidade superficial do que o declive do terreno. Assim, marcas de preparo do solo, direcionadas à pendente do terreno, aumentam a rugosidade e diminuem a capacidade do solo para armazenar água na superfície, em relação à rugosidade ao acaso.

A rugosidade superficial do solo diminuiu com o aumento das chuvas simuladas (Tabela 1), de acordo com o que 
constataram, também, Eltz \& Norton (1997), Bertol et al. (2006) e Castro et al. (2006). A maior redução ocorreu no tratamento sem resíduo (T1) e, a menor, no tratamento com a maior dose de resíduo (T5). Assim, no T1, a redução da rugosidade superficial, pelo efeito das chuvas, foi de $31 \%$ para os dados originais, $45 \%$ para os dados sem o efeito do declive do terreno, e $42 \%$ para os dados ao acaso, enquanto no $\mathrm{T} 5$, as referidas reduções foram, respectivamente, de $35 \%, 36 \%$ e $26 \%$. Estes dados demonstram que o resíduo vegetal de milho - parte do qual mantida na superfície, outra parte semi-incorporada e outra incorporada ao solo - apresentou efeito positivo na preservação da rugosidade superficial, quando esta foi submetida às chuvas.

A tortuosidade superficial do solo pode indicar a dificuldade que a enxurrada terá para escoar, pode indicar o grau de turbulência que a massa de água sofrerá durante o escoamento e, principalmente, a capacidade de armazenagem superficial do solo.

Após o preparo do solo, a tortuosidade superficial foi semelhante à obtida por Eltz \& Norton (1997) e Bertol et al. (2006), tendo variado com os tratamentos e com o volume de chuvas simuladas aplicadas (Tabela 2). O preparo do solo aumentou a tortuosidade ao acaso em $15 \%$, em relação à ausência de preparo, na média dos tratamentos. Este incremento de 1,038 para 1,194 na tortuosidade, embora pareça pequeno, representa um importante aumento na dificuldade para a água escoar na superfície do solo. Além disso, representa um expressivo aumento da capacidade de armazenagem superficial de água pelo solo. Considerando-se o efeito do preparo do solo no tratamento sem resíduo (T1), o aumento da tortuosidade ao acaso, em relação à rugosidade anterior ao preparo foi de $25 \%$, enquanto na média dos tratamentos com resíduo, o referido aumento foi de $12 \%$. A porção do resíduo não incorporado ao solo, pelo preparo, diminuiu a tortuosidade superficial em relação ao solo sem resíduo, justificado do mesmo modo como para a rugosidade. Este efeito foi mais pronunciado no tratamento com a maior dose de resíduo aplicado ao solo (T5).

A tortuosidade superficial, calculada com os dados originais, foi $4 \%$ maior do que a tortuosidade ao acaso, na

Tabela 1. Rugosidade $(\mathrm{mm})$ da superfície do solo com doses de resíduo de milho, submetido ao preparo e às chuvas simuladas com intensidade de $65 \mathrm{~mm} \mathrm{~h}^{-1}$ e duração de uma hora (70,4 mm cada chuva).

\begin{tabular}{|c|c|c|c|c|c|}
\hline \multirow{2}{*}{$\begin{array}{l}\text { Momento da } \\
\text { determinação }\end{array}$} & \multicolumn{5}{|c|}{ Tratamento $^{(1)}$} \\
\hline & 1 & 2 & 3 & 4 & 5 \\
\hline & \multicolumn{5}{|c|}{ Rugosidade original } \\
\hline Antes preparo ${ }^{(2)}$ & 7,39 & 8,19 & 8,31 & 9,25 & 9,92 \\
\hline Após o preparo & 46,97 & 40,64 & 40,84 & 33,31 & 37,25 \\
\hline Após a $1^{\mathrm{a}}$ chuva & 41,46 & 39,75 & 38,43 & 34,79 & 29,45 \\
\hline Após a $2^{\mathrm{a}}$ chuva & 37,68 & 37,16 & 38,60 & 33,34 & 29,28 \\
\hline Após a $4^{\mathrm{a}}$ chuva & 36,82 & 34,99 & 35,03 & 32,69 & 27,61 \\
\hline Após a $6^{\mathrm{a}}$ chuva & 36,52 & 37,88 & 35,75 & 32,70 & 28,19 \\
\hline Após a $8^{\mathrm{a}}$ chuva & 32,27 & 35,30 & 34,94 & 31,25 & 24,30 \\
\hline \multirow[t]{2}{*}{ Média } & 38,62 & 37,62 & 31,31 & 33,00 & 29,35 \\
\hline & \multicolumn{5}{|c|}{ Rugosidade linear } \\
\hline Antes preparo ${ }^{(2)}$ & 5,33 & 6,45 & 7,54 & 8,11 & 8,78 \\
\hline Após o preparo & 30,44 & 21,07 & 27,07 & 22,48 & 22,55 \\
\hline Após a $1^{\mathrm{a}}$ chuva & 21,30 & 17,12 & 23,92 & 19,28 & 18,58 \\
\hline Após a $2^{\mathrm{a}}$ chuva & 21,38 & 16,79 & 22,07 & 17,50 & 16,72 \\
\hline Após a $4^{\mathrm{a}}$ chuva & 17,92 & 15,76 & 19,62 & 18,56 & 15,39 \\
\hline Após a $6^{\mathrm{a}}$ chuva & 17,63 & 14,40 & 18,38 & 16,89 & 14,13 \\
\hline Após a $8^{\mathrm{a}}$ chuva & 16,78 & 14,33 & 17,42 & 16,92 & 14,49 \\
\hline \multirow[t]{2}{*}{ Média } & 20,91 & 16,58 & 21,41 & 18,61 & 16,98 \\
\hline & \multicolumn{5}{|c|}{ Rugosidade ao acaso } \\
\hline Antes preparo ${ }^{(2)}$ & 4,14 & 5,93 & 6,76 & 7,16 & 7,80 \\
\hline Após o preparo & 24,57 & 15,71 & 18,20 & 18,05 & 15,32 \\
\hline Após a $1^{-}$chuva & 17,22 & 11,98 & 16,01 & 14,72 & 13,65 \\
\hline Após a $2^{\mathrm{a}}$ chuva & 16,92 & 11,86 & 15,32 & 13,14 & 12,55 \\
\hline Após a $4^{\mathrm{a}}$ chuva & 14,53 & 10,66 & 13,82 & 13,43 & 11,35 \\
\hline Após a $6^{\mathrm{a}}$ chuva & 14,36 & 10,29 & 13,10 & 12,39 & 11,01 \\
\hline Após a $8^{-}$chuva & 14,26 & 10,23 & 12,77 & 12,34 & 11,32 \\
\hline Média & 16,98 & 11,79 & 14,87 & 14,01 & 12,53 \\
\hline
\end{tabular}

(1) 1: sem resíduo; 2: $2 \mathrm{t} \mathrm{ha}^{-1}$ de resíduo; 3: 4 t ha-1 de resíduo; 4: $6 \mathrm{t} \mathrm{ha-1}$ de resíduo; 5: 8 t ha-1 de resíduo. ${ }^{(2)}$ Excluído da média. 
média dos tratamentos (Tabela 2), o que concorda com dados obtidos por Eltz \& Norton (1997) e Bertol et al. (2006). Isto significa que, ao contrário do que ocorreu com a rugosidade ao acaso, a declividade do terreno de $12 \% \mathrm{e}$ as marcas do preparo do solo pouco influenciaram a tortuosidade ao acaso.

A retirada do efeito da declividade do terreno, no cálculo da tortuosidade, manteve o seu valor $4 \%$ maior do que aquele obtido com os dados ao caso (Tabela 2); este fato indica que a declividade praticamente não teve efeito sobre a tortuosidade da superfície do solo, contrariamente ao que ocorreu com a rugosidade. Marcas de preparo do solo, mesmo direcionadas à pendente do terreno, não aumentam a tortuosidade superficial, mas, efetivamente, diminuem a capacidade do solo para armazenar água na superfície, em relação à rugosidade ao acaso.

As doses do resíduo cultural de milho semi-incorporado influenciaram a rugosidade ao acaso da superfície do solo (Figura 1). Na ausência de resíduo, o coeficiente de decaimento da rugosidade foi de 0,00094 , o que significa que esta se reduziu $0,01636 \mathrm{~mm}$ para cada milímetro de chuva simulada aplicada. No tratamento com $2 \mathrm{t} \mathrm{ha}^{-1}$ de resíduo, o referido coeficiente foi de 0,00071 , equivalente à redução média de $0,00856 \mathrm{~mm}$ da rugosidade para cada milímetro de chuva. O decaimento foi de 0,00063 para o tratamento com 4 t ha ${ }^{-1}$ de resíduo, o que equivale à redução média de $0,00956 \mathrm{~mm}$ da rugosidade para cada milímetro de chuva. Para o tratamento com $6 \mathrm{t} \mathrm{ha}^{-1}$ de resíduo, o coeficiente foi de 0,00061 , equivalente à redução média de $0,00891 \mathrm{~mm}$ da rugosidade para cada milímetro de chuva e, no tratamento com 8 t ha $^{-1}$ de resíduo, o decaimento foi de 0,00058 , que significou uma redução média de $0,00740 \mathrm{~mm}$ da rugosidade para cada milímetro de chuva simulada aplicada.

Assim, a redução da rugosidade ao acaso, por milímetro de chuva simulada, foi 1,9 vez maior no solo sem resíduo $(0,01636 \mathrm{~mm})$ do que na média dos tratamentos com resíduo $(0,00862 \mathrm{~mm})$, o que revela a importância do resíduo vegetal na preservação da rugosidade superficial do solo.

A expectativa de redução da rugosidade ao acaso, pelo efeito da chuva, é indicada pelo grau de ajuste do modelo exponencial utilizado, expresso pelo coeficiente de determinação. Isto mostra que a rugosidade superficial

Tabela 2. Tortuosidade (adimensional) da superfície do solo com doses de resíduo de milho, submetido ao preparo e às chuvas simuladas com intensidade de $65 \mathrm{~mm} \mathrm{~h}^{-1}$ e duração de uma hora (70,4 mm cada chuva).

\begin{tabular}{|c|c|c|c|c|c|}
\hline \multirow{2}{*}{$\begin{array}{l}\text { Momento da } \\
\text { determinação }\end{array}$} & \multicolumn{5}{|c|}{ Tratamento $^{(1)}$} \\
\hline & 1 & 2 & 3 & 4 & 5 \\
\hline & \multicolumn{5}{|c|}{ Tortuosidade original } \\
\hline Antes do preparo ${ }^{(2)}$ & 1,011 & 1,031 & 1,041 & 1,051 & 1,065 \\
\hline Após o preparo & 1,316 & 1,181 & 1,265 & 1,209 & 1,222 \\
\hline Após a $1^{\mathrm{a}}$ chuva & 1,171 & 1,122 & 1,212 & 1,148 & 1,172 \\
\hline Após a $2^{\mathrm{a}}$ chuva & 1,153 & 1,112 & 1,167 & 1,129 & 1,140 \\
\hline Após a $4^{-a}$ chuva & 1,113 & 1,088 & 1,137 & 1,125 & 1,098 \\
\hline Após a $6^{\mathrm{a}}$ chuva & 1,106 & 1,074 & 1,123 & 1,097 & 1,098 \\
\hline Após a $8^{\text {a }}$ chuva & 1,095 & 1,070 & 1,111 & 1,099 & 1,111 \\
\hline \multirow[t]{2}{*}{ Média } & 1,159 & 1,108 & 1,169 & 1,135 & 1,140 \\
\hline & \multicolumn{5}{|c|}{ Tortuosidade linear } \\
\hline Antes do preparo ${ }^{(2)}$ & 1,011 & 1,031 & 1,041 & 1,051 & 1,064 \\
\hline Após o preparo & 1,316 & 1,181 & 1,265 & 1,209 & 1,221 \\
\hline Após a $1^{\underline{a}}$ chuva & 1,171 & 1,122 & 1,212 & 1,148 & 1,173 \\
\hline Após a $2^{\mathrm{a}}$ chuva & 1,152 & 1,112 & 1,167 & 1,129 & 1,140 \\
\hline Após a $4^{\mathrm{a}}$ chuva & 1,112 & 1,087 & 1,137 & 1,126 & 1,098 \\
\hline Após a $6^{\mathrm{a}}$ chuva & 1,106 & 1,074 & 1,123 & 1,097 & 1,098 \\
\hline Após a $8^{\mathrm{a}}$ chuva & 1,095 & 1,068 & 1,111 & 1,099 & 1,111 \\
\hline \multirow[t]{2}{*}{ Média } & 1,159 & 1,107 & 1,169 & 1,135 & 1,140 \\
\hline & \multicolumn{5}{|c|}{ Tortuosidade ao acaso } \\
\hline Antes do preparo ${ }^{(2)}$ & 1,010 & 1,030 & 1,039 & 1,048 & 1,063 \\
\hline Após o preparo & 1,261 & 1,158 & 1,193 & 1,169 & 1,164 \\
\hline Após a $1^{\underline{a}}$ chuva & 1,132 & 1,092 & 1,156 & 1,117 & 1,134 \\
\hline Após a $2^{\mathrm{a}}$ chuva & 1,123 & 1,085 & 1,125 & 1,099 & 1,115 \\
\hline Após a $4^{\text {a }}$ chuva & 1,090 & 1,066 & 1,098 & 1,091 & 1,076 \\
\hline Após a $6^{\mathrm{a}}$ chuva & 1,084 & 1,056 & 1,086 & 1,076 & 1,084 \\
\hline Após a $8^{\text {a }}$ chuva & 1,079 & 1,051 & 1,086 & 1,076 & 1,093 \\
\hline Média & 1,128 & 1.085 & 1,124 & 1,105 & 1,111 \\
\hline
\end{tabular}

(1) 1: sem resíduo; 2: $2 \mathrm{tha}^{-1}$ de resíduo; 3: $4 \mathrm{tha} \mathrm{a}^{-1}$ de resíduo; 4: $6 \mathrm{tha}{ }^{-1}$ de resíduo; 5: $8 \mathrm{t} \mathrm{ha}{ }^{-1}$ de resíduo. ${ }^{(2)}$ Excluído da média. 
decaiu diferentemente nos diversos tratamentos. O coeficiente de decaimento da rugosidade, claramente, diminuiu com o aumento da dose de resíduo, enquanto a efetiva rugosidade apresentou apenas uma tendência de diminuição, o que revela a influência de outras variáveis, na redução da rugosidade ocasionada pela chuva, não controladas no experimento. Entre essas variáveis, pode-se citar a porcentagem de cobertura do solo, ocasionada pelo resíduo, e as propriedades físicas, especialmente a estrutura do solo, as quais devem ter variado entre os tratamentos.

O modelo exponencial utilizado ajustou-se aos dados de rugosidade ao acaso influenciada pela chuva, e apresentou coeficientes de determinação altos e significativos, de 0,665 a 0,896 (Figura 1), o que concorda com o tipo de correlação obtida por Bertol et al. (2006). O decaimento exponencial da rugosidade ao acaso da superfície do solo, pelo efeito da chuva, ao longo do tempo, é um comportamento lógico, significando que a rugosidade diminui mais rapidamente por ocasião das primeiras chuvas do que das últimas. Normalmente, o decaimento da rugosidadeé mais acentuado no período de tempo compreendido entre o início da chuva e o início da enxurrada, como ocorreu neste trabalho e que foi, também, constatado por Eltz \& Norton (1997).

A tortuosidade ao acaso da superfície do solo foi influenciada pelas doses do resíduo cultural de milho semiincorporado ao solo (Figura 2), de maneira distinta do que

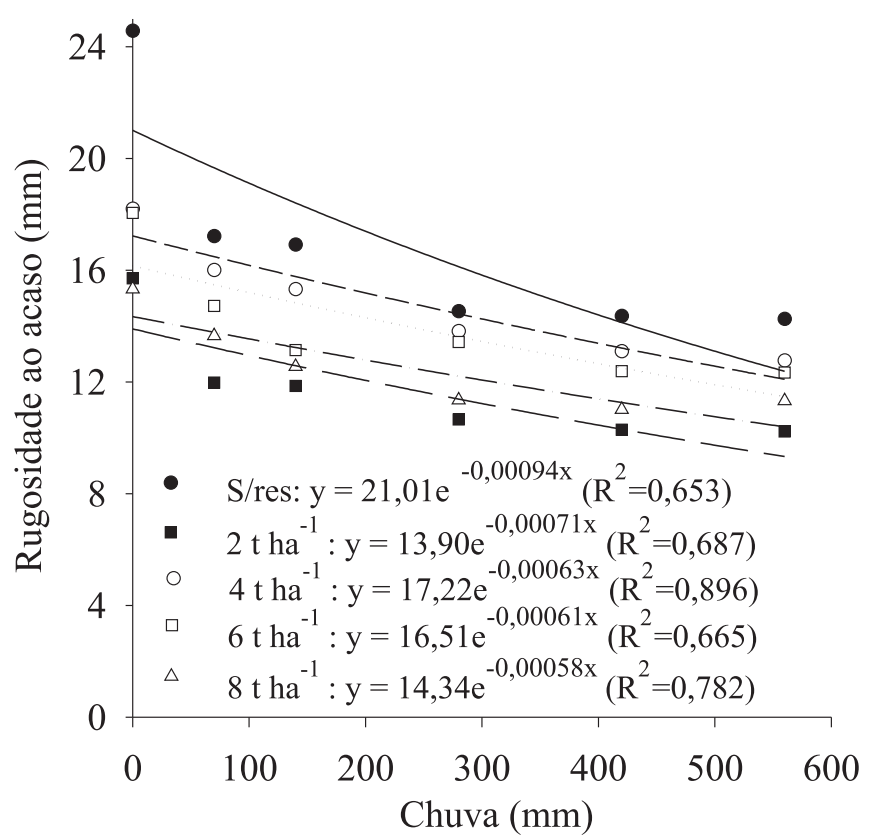

Figura 1. Relação exponencial entre a rugosidade ao acaso da superfície do solo e o volume de chuva simulada, em solo preparado com distintas doses de resíduo cultural de milho. ocorreu com a rugosidade (Figura 1). Os valores dos coeficientes de decaimento indicam que a rugosidade decresceu cerca de 4,5 vezes mais do que a tortuosidade. Isto é explicado pelo fato de que a rugosidade representa a variação vertical do microrrelevo superficial, enquanto a tortuosidade representa a variação horizontal. Assim, o rebaixamento das cristas das microelevações, em decorrência da desagregação do solo pela ação da chuva, faz com que o solo desagregado se deposite nas microdepressões, o que se reflete rápida e intensamente na redução do índice de rugosidade e, menos intensamente na redução da tortuosidade superficial. O grau de ajuste do modelo exponencial aos dados de tortuosidade dos diversos tratamentos foi bom, pois os coeficientes de determinação foram significativos e variaram de 0,611 a 0,825 .

$\mathrm{O}$ valor do coeficiente de decaimento da tortuosidade ao acaso, para o solo sem resíduo $(-0,00024)$, foi 1,8 vez maior do que o valor médio dos tratamentos com resíduo de milho (-0,000135) (Figura 2), de modo semelhante ao que ocorreu com a rugosidade, o que significa uma diferença de redução da tortuosidade de 0,02216 , entre o solo sem resíduo e os demais tratamentos. Isto demonstra a importância do efeito do resíduo cultural de milho semiincorporado ao solo na preservação da tortuosidade.

A eliminação do efeito da declividade do terreno, no procedimento de cálculo da rugosidade (rugosidade linear,

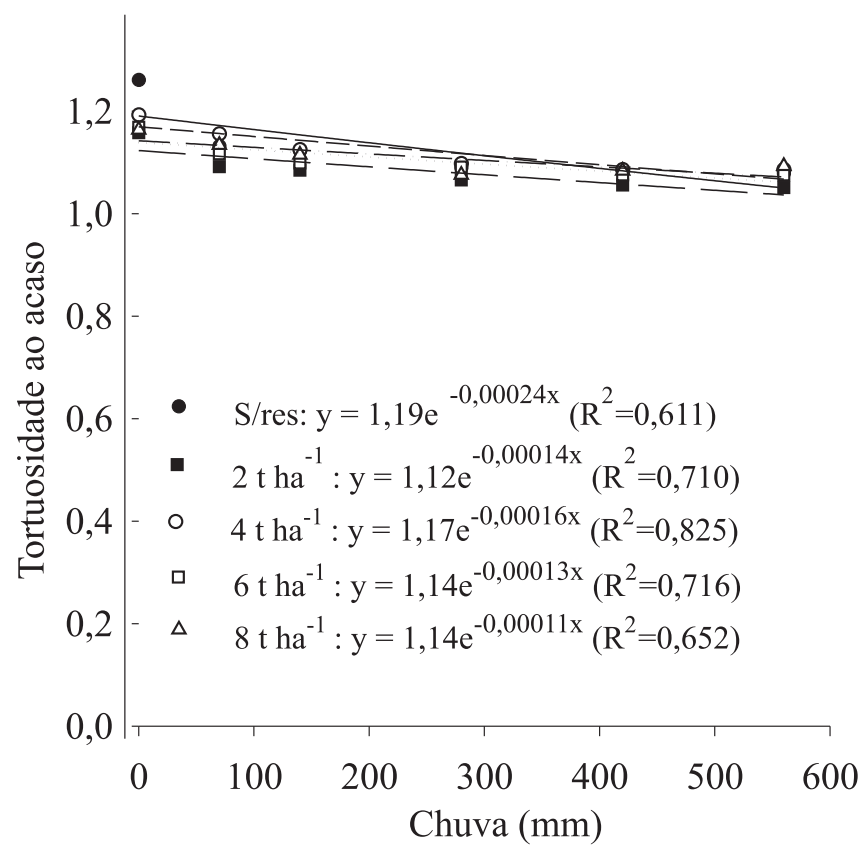

Figura 2. Relação exponencial entre a tortuosidade ao acaso da superfície do solo e o volume de chuva simulada, em solo preparado com distintas doses de resíduo cultural de milho. 
Figura 3 a), afetou menos o índice de rugosidade do que a eliminação conjunta da declividade do terreno e das marcas do preparo do solo, para a obtenção da rugosidade ao acaso (Figura 3 b), o que foi demonstrado pelos coeficientes de inclinação do modelo linear, utilizado para o ajuste às variáveis. Assim, a variação de um milímetro da rugosidade original ocasionou variação de $0,461 \mathrm{~mm}$ da rugosidade linear (Figura 3 a), enquanto a variação de um milímetro da rugosidade linear ocasionou variação de $0,696 \mathrm{~mm}$ da rugosidade ao acaso (Figura $3 \mathrm{~b}$ ). Isto significa que a rugosidade superficial do solo é mais fortemente influenciada pelas marcas do preparo do solo provocadas pelos implementos agrícolas do que pela declividade do terreno.

A tortuosidade foi mais influenciada, quando se eliminou a declividade do terreno no procedimento de cálculo, do que quando se eliminaram, conjuntamente, a declividade do
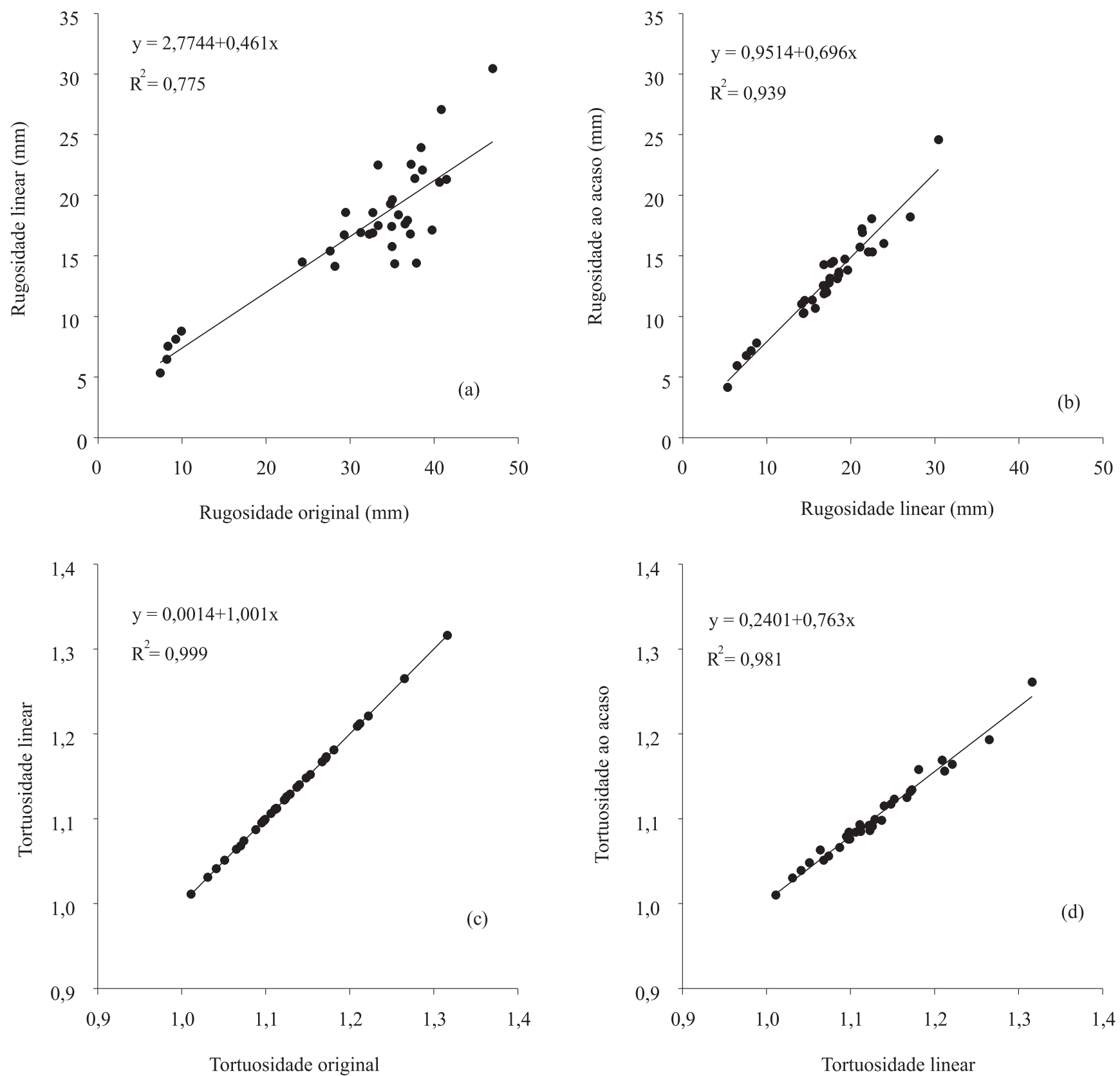

Figura 3. Relação linear entre variáveis da superfície do solo preparado com distintas doses de resíduo cultural de milho. 
terreno e as marcas do preparo do solo (Figura $3 \mathrm{c}$ e d), o que foi demonstrado pelos coeficientes de inclinação do modelo linear, utilizado para o ajuste às variáveis. Assim, a variação da unidade de tortuosidade original (Figura $3 \mathrm{c}$ ) ocasionou variação da unidade de tortuosidade linear, enquanto a variação da unidade de tortuosidade linear (Figura 3 d) ocasionou a variação de 0,763 unidade de tortuosidade ao acaso. Portanto, a tortuosidade superficial do solo, diferentemente da rugosidade, é mais fortemente influenciada pela declividade do terreno do que pelas marcas do preparo do solo provocadas pelos implementos agrícolas.

\section{Conclusões}

1. A rugosidade e a tortuosidade ao acaso, da superfície do solo, são menores do que a rugosidade e a tortuosidade linear e, estas, menores do que a rugosidade e a tortuosidade originais.

2. A redução da rugosidade e da tortuosidade da superfície do solo, em decorrência da aplicação de chuvas simuladas, é influenciada pela dose do resíduo cultural de milho semi-incorporado ao solo pelo preparo.

3. O decaimento da rugosidade ao acaso da superfície do solo, pelo efeito de chuvas simuladas, diminui com o aumento da dose de resíduo cultural de milho semiincorporado ao solo pelo preparo; no caso da tortuosidade ao acaso, há apenas tendência de redução, com o aumento da dose do resíduo de $2 \mathrm{t} \mathrm{ha} \mathrm{a}^{-1}$ para $8 \mathrm{t} \mathrm{ha}^{-1}$.

4. A rugosidade superficial do solo é mais fortemente influenciada quando se elimina o efeito da declividade do terreno do que quando se eliminam, simultaneamente, os efeitos da declividade do terreno e das marcas de preparo do solo; para o caso da tortuosidade, quando se eliminam, conjuntamente, a declividade do terreno e as marcas de preparo do solo, o efeito é maior do que quando se elimina apenas o efeito da declividade.

\section{Agradecimentos}

Ao CNPq, Brasil, e à Universidad de A Coruña, Espanha, pelo apoio financeiro.

\section{Referências}

BERTOL, I.; AMARAL, A.J.; VIDAL VÁZQUEZ, E.; PAZ GONZÁLEZ, A.; BARBOSA, F.T. Relações da rugosidade superficial do solo com o volume de chuva e com a estabilidade de agregados em água. Revista Brasileira de Ciência do Solo, v.30, p.543-553, 2006.

BERTOL, I.; COGO, N.P.; LEVIEN, R. Erosão hídrica em diferentes preparos do solo logo após as colheitas de milho e trigo, na presença e na ausência dos resíduos culturais. Revista Brasileira de Ciência do Solo, v.21, p.409-418, 1997.

CASTRO, L.G.; COGO, N.P.; VOLK, L.B.S. Alterações na rugosidade superficial do solo pelo preparo e pela chuva e sua relação com a erosão hídrica. Revista Brasileira de Ciência do Solo, v.30, p.339-352, 2006.

DARBOUX, F.; HUANG, C. Does soil surface roughness increase or decrease water and particle transfers? Soil Science Society of America Journal, v.69, p.748-756, 2005.

ELTZ, F.L.F.; NORTON, L.D. Surface roughness changes as affected by rainfall erosivity, tillage and canopy cover. Soil Science Society of America Journal, v.61, p.1746-1755, 1997.

HUANG, C.; BRADFORD, J.M. Application of a laser scanner to quantify soil microtopography. Soil Science Society of America Journal, v.56, p.14-21, 1992.

KAMPHORST, E.C.; JETTEN, V.; GUÉRIF, J.; PITKÄNEN, J.; IVERSEN, B.V.; DOUGLAS, J.T.; PAZ, A. Predicting depressional storage from soil surface roughness. Soil Science Society of America Journal, v.64, p.1749-1758, 2000.

LEHRSCH, G.A.; WHISLER, F.D.; RÖMKENS, M.J.M. Soil surface roughness as influenced by selected soil physical properties. Soil and Tillage Research, v.10, p.197-212, 1987.

LINDEN, D.R.; VAN DOREN JUNIOR, D.M. Parameters for characterizing tillage-induced soil surface roughness. Soil Science Society of America Journal, v.50, p.1560-1565, 1986.

NAVAS, A.; ALBERTO, F.; MACHIN, J.; GALAN, A. Design and operation of a rainfall simulator for field studies of runoff and soil erosion. Soil Technology, v.3, p.385-397, 1990.

ONSTAD, C.A. Depressional storage on tilled soil surfaces. Transactions of the American Society of Agricultural Engineers, v.27, p.729-732, 1984.

PAZ GONZÁlEZ, A.; TABOADA CASTRO, M.T. Medida del microrrelieve del suelo y estimación de la retención hídrica en depresiones de la superficie. Cadernos do Laboratorio Xeolóxico de Laxe, v.21, p.829-842, 1996.

RENARD, K.G.; FOSTER, G.R.; WEESIES, G.A.; McCOOL, D.K.; YODER, D.C. Predicting soil erosion by water: a guide to conservation planning with the revised universal soil loss equation (RUSLE). Washington: U.S. Department of Agriculture, 1997.384p. (Agriculture handbook, 703)

RÖMKENS, M.J.M.; WANG J.Y. Effect of tillage on soil roughness. Transactions of the American Society of Agricultural Engineers, v.29, p.429-433, 1986.

SKIDMORE, E.L. Comments on chain method for measuring soil roughness. Soil Science Society of America Journal, v.61, p.15321533, 1997.

Recebido em 3 de maio de 2006 e aprovado em 24 de outubro de 2006 